Bull. Austral. Math. Soc.

VOL. 49 (1994) [519-521]

\title{
A REMARK ON EMBEDDING TOPOLOGICAL GROUPS INTO PRODUCTS
}

\author{
VLADIMIR PESTOV
}

\begin{abstract}
Let $\mathcal{P}$ be a class of topological groups such that every topological group is isomorphic to a topological subgroup of the direct product (with Tychonoff topology) of a subfamily of $\mathcal{P}$. Then every Tychonoff space is homeomorphic to a subspace of a group from $\mathcal{P}$.
\end{abstract}

QUESTION. Let $\mathcal{P}$ be a certain class of topological groups. Is every topological group isomorphic to a topological subgroup of the direct product (with the Tychonoff topology) of a subfamily of $\mathcal{P}$ ?

This question was discussed for various classes of topological groups $\mathcal{P}$ by Arhangel'skiĭ $[1,2]$. In particular, it was answered in the negative independently by the author [8] and Guran [4] in the case where $\mathcal{P}$ was the class of all topological groups with unity of type $G_{\delta}$, and also by Guran [5] in the case where $\mathcal{P}$ was the class of all topological groups whose underlying topological spaces were sequential.

The following result throws new light on all possible questions of this kind.

Theorem. Let $\mathcal{P}$ be a class of topological groups. Suppose that continuous homomorphisms to the groups from $\mathcal{P}$ separate points in every Hausdorff topological group $G$. Then every Tychonoff space is homeomorphic to a subspace of a group from $\mathcal{P}$.

Proof: Let $X$ be an arbitrary Tychonoff space. One can assume without loss in generality that $X$ is compact. It can be embedded into a Tychonoff space $Y$ such that any ordered $n$-tuple of pairwise distinct elements of $Y$ can be sent to any other such $n$-tuple by means of an autohomeomorphism of $Y$ [7]. The full autohomeomorphism group, Aut $Y$, of $Y$ acts on the free topological group, $F(Y)$, on $Y$ [6] if one extends autohomeomorphisms of $Y$ to automorphisms of $F(Y)$. This action is continuous if

Received 25th August, 1993

This work was done during a visit of the author to the Analysis Research Group of the University of Wollongong in January 1993, and the author is thankful to Professor Sidney Morris for his kind hospitality. A Small Research Grant V212/451/RGNT/594/153 from the Internal Grants Committee of the Victoria University of Wellington is acknowledged.

Copyright Clearance Centre, Inc. Serial-fee code: 0004-9729/94 \$A2.00+0.00. 
the group Aut $Y$ is endowed with the discrete topology. Form the semidirect product $G=$ Aut $Y \propto F(Y)$.

It follows from the assumption of the Theorem that here exist an $H \in \mathcal{P}$ and a continuous $f: G \rightarrow H$ such that $f(Y)$ is non-trivial. The restriction of $f$ to $Y$ is one-toone. (Indeed, otherwise there exist pairwise distinct $x, y, z \in Y$ such that $f(x)=f(y) \neq$ $f(z)$. There is an $h \in$ Aut $Y$ with $h^{-1} x h=y, h^{-1} y h=z$, and $h^{-1} z h=x$. One has $f(y)=f(h)^{-1} f(x) f(h)=f(h)^{-1} f(y) f(h)=f(z)$, a contradiction.) Therefore, $\left.f\right|_{X}$ is a homeomorphism by virtue of the compactness of $X$.

COROLlary. Let $\mathcal{P}$ be a class of topological groups. Suppose that every Hausdorff topological group $G$ is isomorphic to a topological subgroup of the direct product (with the Tychonoff topology) of a subfamily of $\mathcal{P}$. Then every Tychonoff space is homeomorphic to a subspace of a group from $\mathcal{P}$.

In particular, the two cases mentioned above receive simple answers in the negative.

We hope that our remark can be put in the context of generating varieties of topological groups [3]. In particular, we suggest the following.

ConjeCture. Let $\mathcal{P}$ be a class of topological groups. The following are equivalent:

(i) every Hausdorff topological group $G$ is isomorphic to a topological subgroup of the direct product (with the Tychonoff topology) of a subfamily of $\mathcal{P}$;

(ii) every Hausdorff topological group $G$ is isomorphic to a topological subgroup of a group from $\mathcal{P}$.

\section{REFERENCES}

[1] A.V. Arhangel'skii, 'Relations among invariants of topological groups and their subspaces', Russian Math. Surveys 35 (1980), 1-23.

[2] A.V. Arhangel'skiǐ, 'Classes of topological groups', Russian Math. Surveys 36 (1981), 151-174.

[3] M.S. Brooks, S.A. Morris and H.B. Thompson, 'Generating varieties of topological groups', Proc. Edinbourgh Math. Soc. 18 (1973), 191-197.

[4] I.I. Guran, 'Topology of an infinite symmetric group and condensations', (in Russian), Comment. Math. Univ. Carolin. 22 (1981), 311-316.

[5] I.I. Guran, A private communication, 1992.

[6] A.A. Markov, 'Three papers on topological groups', Amer. Math. Soc. Transl. 30 (1950).

[7] M.G. Megrelishvili, 'On the imbedding of topological spaces into spaces with strong properties of homogenuity', (in Russian), Bull. Acad. Sci. Georgian SSR 121 (1986), 257-260.

[8] V.G. Pestov, 'On embeddings and condensations of topological groups', Math. Notes 31 (1982), 228-230. 
Department of Mathematics

Victoria University of Wellington

Wellington

New Zealand

e-mail: vladimir.pestov@vuw.ac.nz 